\title{
DISCONTINUOUS INTEGRALS AND GENERALIZED POTENTIAL THEORY
}

\author{
BY \\ ALEXANDER WEINSTEIN
}

1. Introduction. The main purpose of this paper is to give asymptotic formulae for certain integrals involving products of Bessel's functions. In particular the paper gives a new and elementary approach to the theory of the famous Weber-Schafheitlin discontinuous integrals which have been investigated by several generations of mathematicians. Up to now the evaluation of these integrals has been done, according to Titchmarsh, by a delicate application of Mellin's transformation with tedious details $\left({ }^{1}\right)$.

The new approach is based on a generalized potential theory in a fictitious space of $n$ dimensions, for $n>2$, where $n$ is not necessarily an integral number. The computation of the integrals is reduced to a simple application of the divergence theorem in a meridian plane of this space. This gives a simple interpretation of the results which, as we shall see, could easily be guessed from the analogy to the ordinary three-dimensional space.

As an important by-product, we obtain explicit formulas for the fundamental solutions of a class of partial differential equations which we call the generalized Stokes-Beltrami equations.

2. The generalized Stokes-Beltrami equations. In the following we shall use three constants $n, p$, and $q$ related by the equations

$$
n=p+2, \quad p=2 q+1
$$

where $n>2, p>0, q>-1 / 2$.

Let $\phi(x, y)$ and $\psi(x, y)$ be a pair of functions which possess in the halfplane $y>0$ continuous second derivatives and satisfy the following differential equations:

$$
\begin{aligned}
& y^{p} \frac{\partial \phi}{\partial x}=\frac{\partial \psi}{\partial y} \\
& y^{p} \frac{\partial \phi}{\partial y}=-\frac{\partial \psi}{\partial x} .
\end{aligned}
$$

Presented to the Society, February 23, 1946, under the title On Stokes' stream function and Weber's discontinuous integral; received by the editors February 14, 1947.

(1) E. C. Titchmarsh, Introduction to the theory of Fourier integrals, Oxford, 1937, p. 202. See also the formidable computations in G. N. Watson, Theory of Bessel functions, Cambridge, 1922, especially pp. 399-405. 
These equations have been considered for $p=1$ by Stokes and Beltrami $\left({ }^{2}\right)$, and for any positive values of $p$ by Bers and Gelbart $\left({ }^{3}\right)$. In the case $p=1, \phi(x, y)$ represents the values taken by an axially symmetric harmonic function (or potential) $\Phi(x, y, z)$ in the meridian plane $(x, y)$ of the three-dimensional space, $x$ being the axis of symmetry. The associated function $\psi(x, y)$ is called the Stokes stream function.

For any positive value of $p$, we shall adopt the same nomenclature and call $\phi(x, y)$ in (1) an axially symmetric potential and $\psi(x, y)$ its associated stream function in a fictitious "space" of $n=p+2=3+2 q$ dimensions, with a "meridian plane" $(x, y)$ :

From (1) follows

$$
\begin{aligned}
& y\left(\phi_{x x}+\phi_{y y}\right)+p \phi_{y}=0, \\
& y\left(\psi_{x x}+\psi_{y y}\right)-p \psi_{y}=0 .
\end{aligned}
$$

The system (1) and each of the equations (2) and (3) are equivalent. The equations (2) and (3) can also be written as follows:

$$
\begin{aligned}
\operatorname{div}\left(y^{p} \operatorname{grad} \phi\right) & =0, \\
\operatorname{div}\left(y^{-p} \operatorname{grad} \psi\right) & =0 .
\end{aligned}
$$

The solutions of these elliptic differential equations are analytic for $y>0$.

The $x$-axis is in general a singular line for $\phi(x, y)$. However, some solutions $\phi$ of (2) remain analytic for $y=0$. If, moreover, $\partial \phi / \partial y$ vanishes on the $x$-axis, $\phi$ can be defined for $y<0$. In this case $\phi$ is an even function of $y$.

An important solution of (2) is the function $\phi_{0}(x, y)=r^{-p}=\left(x^{2}+y^{2}\right)^{-p / 2}$, where $r^{2}=x^{2}+y^{2}$.

Denoting by $C$ the boundary of a domain $R$, by $s$ the arclength on $C$, and by $n$ its exterior normal, we have the following Green's formulas for two regular functions $\phi$ and $\phi^{*}$ :

$$
\begin{gathered}
\iint_{R} y^{p}\left(\phi_{x x}+\phi_{y y}+p y^{-1} \phi_{y}\right) d x d y=\int_{C} y^{p} \frac{\partial \phi}{\partial n} d s \\
\iint_{R}\left[\phi^{*} \operatorname{div}\left(y^{p} \operatorname{grad} \phi\right)-\phi \operatorname{div}\left(y^{p} \operatorname{grad} \phi^{*}\right)\right] d x d y \\
=\int_{C} y^{p}\left(\phi^{*} \frac{\partial \phi}{\partial n}-\phi \frac{\partial \phi^{*}}{\partial n}\right) d s .
\end{gathered}
$$

Moreover, if $\phi$ satisfies (2), we have, by (1), for any arc $P Q$ connecting two

(2) E. Beltrami, Opere matematiche, vol. 3, Milano, 1911 (especially pp. 349-382).

(3) L. Bers and A. Gelbart, Quarterly of Applied Mathematics vol. 1 (1943) pp. 168-188, especially p. 176. See also H. Bateman, Partial differential equations of mathematical physics, Cambridge, 1932, p. 408. 
points $P$ and $Q$, denoting here by $n$ the normal in the direction to the right of $P Q$,

$$
\int_{P Q} y^{p} \frac{\partial \phi}{\partial n} d s=\psi(Q)-\psi(P)
$$

Let $\phi$ be an even solution of (2). Taking $\phi^{*}=r^{-p}$, we apply (5) to a halfcircle $x^{2}+y^{2} \leqq a^{2}, y \geqq 0$. Excluding the origin by an infinitesimal half-circle and observing that $\partial \phi / \partial n$ and $\partial \phi^{*} / \partial n$ vanish on the $x$-axis, we obtain the following

Mean value theorem.

$$
\phi(0,0) \int_{0}^{\pi} \sin ^{p} \theta d \theta=\int_{0}^{\pi} \phi(x, y) \sin ^{p} \theta d \theta,
$$

where $\theta$ denotes the polar angle. The integral on the right-hand side is taken over $a$ half-circle of arbitrary radius $a$.

A similar formula holds obviously for $\phi\left(x_{0}, 0\right)$ at any regular point $\left(x_{0}, 0\right)$ of $\phi$.

This mean value theorem leads to the following fundamental identification principle. If two even potentials $\phi_{1}$ and $\phi_{2}$ are regular and identical on a segment $L$ of the $x$-axis, they are identical everywhere. In fact, if the potential $\phi=\phi_{1}-\phi_{2}$ did not vanish identically, there would be a derivative $\partial^{k} \phi / \partial y^{k}$ which would not be zero on a subsegment $l$ of $L$ and which can be assumed positive while $\partial \phi / \partial y, \cdots, \partial^{k-1} \phi / \partial y^{k-1}$ are identically zero. The potential $\phi$ being zero on $l, \phi(x, y)$ would then be positive in a domain adjacent to the segment $l$, in contradiction to $(7)\left({ }^{4}\right)$.

3. The fundamental solution of the equation for the potential. The fundamental solution solution of (2), with a singularity at the origin, is given by

$$
\phi_{0}(x, y)=r^{-p}=\left(x^{2}+y^{2}\right)^{-p / 2},
$$

representing the potential of a point-source.

More generally, $\left[\left(x-x_{0}\right)^{2}+y^{2}\right]^{-p / 2}$ has a singularity at the point $\left(x_{0}, 0\right)$ on the $x$-axis. However, if the singularity is at a point $B: x=0, y=b, b>0$, the fundamental solution corresponds to the potential $\phi_{b}(x, y)$ of a "ring of sources" of radius $b$ and of a constant total strength, independent of the value of $b$. Several expressions can be given for $\phi_{b}$, which can be recognized as equivalent by the identification principle. One of the simplest of these formulas is

(4) Let us observe that our proof of the identification principle is essentially different from that usually given in the ordinary three-dimensional space (cf. Kellogg, Potential theory, Berlin, 1929, p. 255). The reason for this is that, at this stage, we have at our disposal only the fundamental solution with a singularity on the axis of symmetry. 
(9)

$$
\phi_{b}(x, y)=S_{p-1} \int_{0}^{\pi}\left(b^{2}+x^{2}+y^{2}-2 b y \cos \alpha\right)^{-p / 2} \sin ^{p-1} \alpha d \alpha
$$

where

$$
S_{p-1}^{-1}=\int_{0}^{\pi} \sin ^{p-1} \alpha d \alpha=\pi^{1 / 2} \Gamma\left(\frac{p}{2}\right) \Gamma^{-1}\left(\frac{p+1}{2}\right) .
$$

The fact that $\phi_{b}$ is a solution of (2) can be verified by differentiating the integrand and by a subsequent integration by parts of the resulting expression. The case $p=1$ is classical. Let

$$
\omega_{n}=2 \pi^{n / 2} \Gamma^{-1}(n / 2)
$$

denote the "area" of the "unit sphere" in the space of $n=p+2=3+2 q$ dimensions. It is then obvious that

$$
S_{p-1}^{-1}=\omega_{p+1} \omega_{p}^{-1} .
$$

Putting $y=0$ in (9), we obtain

$$
\phi_{b}(x, 0)=\left(b^{2}+x^{2}\right)^{-p / 2} .
$$

It is obvious that, for a fixed $b, \phi_{b}(x, y)$ is an analytic function of $x$ and $y$ for $(x, y) \neq(0, b)$.

Moreover $\phi_{b}(x, y)$ is also an analytic function of $b$ (in fact, of $b, x$ and $y$ ) as long as $(x, y) \neq(0, b)$. This holds for all values of $b \geqq 0$.

It may be easily proved that, for $b>0, \phi_{b}(x, y)$ has a logarithmic singularity at the point $B: x=0, y=b$, so that $\phi_{b}$ is a fundamental solution of (2).

In fact, let us observe that $b^{2}+x^{2}+y^{2}-2 b y \cos \alpha=x^{2}+(y-b)^{2}$ $+4 b y \sin ^{2}(\alpha / 2)$. Putting

$$
\left[x^{2}+(y-b)^{2}\right](4 b y)^{-1}=\epsilon^{2},
$$

we obtain for $\phi_{b}$ the expression

$$
\phi_{b}(x, y)=S_{p-1}(4 b y)^{-p / 2} \int_{0}^{\pi}\left(\epsilon^{2}+\sin ^{2} \frac{\alpha}{2}\right)^{-p / 2} \sin ^{p-1} \alpha d \alpha .
$$

It is clear that the difference between the integral in (15) and the integral

$$
\int_{0}^{\pi} \alpha^{p-1}\left(\epsilon^{2}+4^{-1} \alpha^{2}\right)^{-p / 2} d \alpha
$$

is a regular function of $x$ and $y$ even at the point $x=0, y=b$ (that is, for $\epsilon=0$ ). Putting $\alpha=2 \epsilon \xi$ we obtain, in place of (16), the expression

$$
2^{p} \int_{0}^{\pi(2 \epsilon)} \xi^{p-1}\left(1+\xi^{2}\right)^{-p / 2} d \xi
$$


which is, for $p>0$, clearly equal to

$$
-2^{p} \log \epsilon+R_{1}(x, y)
$$

where $R_{1}(x, y)$ is regular at the point $B$. In this way we obtain from (15) and (16), for small values of $x^{2}+(y-b)^{2}$,

$$
\phi_{b}(x, y)=-S_{p-1}(b y)^{-p / 2} \log \left[x^{2}+(y-b)^{2}\right]^{1 / 2}+R(x, y)
$$

where $R(x, y)$ is regular at the point $x=0, y=b$. The formula (17) shows that $\phi_{b}$ is a fundamental solution of (2).

\section{The fundamental solution in terms of Bessel's functions. Let}

$$
J_{q}(\xi)=2^{-q} \Gamma^{-1}(q+1) \xi^{q}\left[1-2^{-1}(2 q+2)^{-1} \xi^{2}+\cdots\right]
$$

denote the Bessel's function of the first kind and of the index $q>-1 / 2$.

We shall prove that (9) can be replaced by the formula

$$
\phi_{b}(x, y)=C_{q} b^{-q} y^{-q} \int_{0}^{\infty} e^{-|x| t} J_{q}(y t) J_{q}(b t) d t
$$

where

$$
C_{q}={ }^{2 q} \Gamma^{2}(q+1) \Gamma^{-1}(2 q+1) .
$$

For the facts concerning the convergence of the integral in (19) see Watson, loc. cit. footnote 1 . In order to prove (19), let us consider the expression

$$
F_{b}(x, y)=b^{-q} y^{-q} \int_{0}^{\infty} e^{-|x| t} J_{q}(y t) J_{q}(b t) d t .
$$

It can be easily verified that, for $x \neq 0$ and $y \geqq 0, F_{b}$ is a regular solution of (2).

Using (18), we see that

$$
F_{b}(x, 0)=2^{-q} \Gamma^{-1}(q+1) \int_{0}^{\infty} e^{-|x| t}(b t)^{-q} J_{q}(b t) t^{2 q} d t .
$$

This well known expression can be easily evaluated by the identification principle.

In fact, the expression $e^{-|x| t}(b t)^{-a} J_{q}(b t)$ considered as a function of $x$ and $b$ satisfies, for $x \neq 0$ and $b \geqq 0$, the differential equation (2) in which $y$ has been replaced by $b$. The same is therefore true for $F_{b}(x, 0)$. Putting $b=0$, we obtain

$$
F_{0}(x, 0)=2^{-2 q} \Gamma^{-2}(q+1) \int_{0}^{\infty} e^{-|x| t t^{2 q}} d t=2^{-2 q} \Gamma^{-2}(q+1) \Gamma(2 q+1)|x|^{-2 q-1} .
$$

The identification principle yields then for all $b \geqq 0$

$$
F_{b}(x, 0)=2^{-2 q} \Gamma^{-2}(q+1) \Gamma(2 q+1)\left(x^{2}+b^{2}\right)^{-p / 2} \quad(p=2 q+1) .
$$

Using (23) and (20), we see that $C_{q} F_{b}(x, y)$ takes, for $y=0$, the same values, 
namely $\left(x^{2}+b^{2}\right)^{-p / 2}$, as $\phi_{b}(x, y)$ in (9). This fact completes the proof of the formula (19).

Since the integral in (19) is convergent for $x=0, y \neq b$, we have, by Abel's theorem, for $x=0, y \neq b$,

$$
\phi_{b}(0, y)=C_{q} b^{-q} y^{-q} \int_{0}^{\infty} J_{q}(y t) J_{q}(b t) d t .
$$

However, it should be noted that this integral is not absolutely convergent. It is only by comparison with the formula

$$
\phi_{b}(0, y)=S_{p-1} \int_{0}^{\pi}\left(b^{2}+y^{2}-2 b y \cos \alpha\right)^{-p / 2} \sin ^{p-1} \alpha d \alpha
$$

that we see that the right-hand side in (24) is an analytic function of $y$ and $b$ for $y \geqq 0, b \geqq 0$, as long as $y \neq b$.

5. The associated stream function. The expression (19) is particularly adapted for the computation of an explicit integral representation for the associated function $\psi_{b}(x, y)$. This was already noticed, in the case $q=0(n=3)$ by Beltrami who, however, failed to recognize that $\psi_{b}$ is a many-valued function for $b \neq 0$. Beltrami's error has been often repeated in the literature and has been corrected only recently(5).

It follows from the generalized Stokes-Beltrami equations (1) and from the results of $\S 3$ that $\psi_{b}(x, y)$ is a regular function of $x$ and $y$ with the exception of the point $B: x=0, y=b$, which is, as we shall see, a branch point of infinite order.

Generalizing Beltrami's computations, we easily obtain from (1) and (19) the following two integral representations for $\psi_{b}$, valid for positive and for negative values of $x$ respectively:

$$
\begin{aligned}
& \psi_{b}=\psi_{b}^{+}(x, y)=-C_{q} b^{-q} y^{q+1} \int_{0}^{\infty} e^{-x t} J_{q+1}(y t) J_{q}(b t) d t, \quad x>0 . \\
& \psi_{b}=\overrightarrow{\psi_{b}}(x, y)=C_{q} b^{-q} y^{q+1} \int_{0}^{\infty} e^{x t} J_{q+1}(y t) \dot{J_{q}}(b t) d t, \quad x<0 .
\end{aligned}
$$

For $x=0$, the integrals are still convergent and we have, by Abel's theorem,

$$
\begin{aligned}
& \psi_{b}^{+}(0, y)=-C_{q} b^{-q} y^{q+1} \int_{0}^{\infty} J_{q+1}(y t) J_{q}(b t) d t, \\
& \psi_{b}^{-}(0, y)=-\psi_{b}^{+}(0, y) .
\end{aligned}
$$

The integral

(ङ) A. Weinstein. Bull. Amer. Math. Soc. vol. 52 (1946) pp. 240, 431, 823. 


$$
W_{b}(y)=\int_{0}^{\infty} J_{q+1}(y t) J_{q}(b t) d t
$$

which appears in (28) is the Weber-Schafheitlin integral. $W_{b}$ is a discontinuous function of $y$, a fact which will be proved here by an elementary and familiar application of the divergence theorem, which, for $q=0(n=3)$ is the basis of the potential theory in the ordinary three-dimensional space. It will follow from our considerations that $\psi_{b}^{+}$and $\psi_{b}^{-}$represent two different branches of the many-valued function $\psi_{b}$.

Let us take two points, $P$ and $Q$ on the $y$-axis, with $y_{P}<y_{Q}<b$. By (6) we have, integrating along the curve $P P_{0} Q_{0} Q$ (Fig. 1):

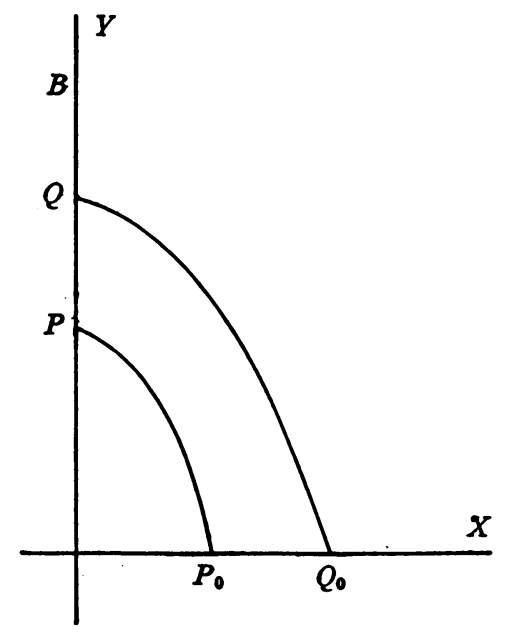

FIG. 1

$$
\int y^{2 q+1} \frac{\partial \phi_{b}}{\partial n} d s=\psi_{b}^{+}(Q)-\psi_{b}^{+}(P) .
$$

Since, on $Q P, \partial \phi_{b} / \partial n$ is zero, the integral can be taken over the closed curve $P P_{0} Q_{0} Q P$. By (4), this integral is zero, which shows that $\psi_{b}^{+}(Q)=\psi_{b}^{+}(P)$.

Denoting by $k$ a constant, we have therefore

$$
\psi_{b}^{+}(0, y)=k
$$

for $y<b$.

For the same reason, we have

$$
\psi_{b}^{+}(0, y)=K
$$$$
\text { for } b<y \text {, }
$$

where $K$ denotes also a constant. Let us also note that by (26) and (28)

$$
\psi_{b}^{+}(x, 0)=0 \quad \text { for } b \neq 0 \text { and } 0 \leqq x<\infty .
$$


In order to determine $k$, we use the fact, mentioned above, that $\psi_{b}^{+}(0, y)$ is continuous in $y$ in the interval $0 \leqq y<b$, so that $k=\psi_{b}^{+}(0,0)$, that is, by (34),

$$
k=0 \text {. }
$$

In view of (32), (28) and (30), we have therefore

$$
W_{b}(y)=0 \quad \text { for } 0 \leqq y<b .
$$

The value of the constant $K$ will be found in the next paragraph as a special case of a general result.

6. The branch point $B$. We prove here the following fundamental theorem: Let the point $Q=(x, y)$ tend to the point $B=(0, b)$ on a smooth curve in such a way that (Fig. 2)

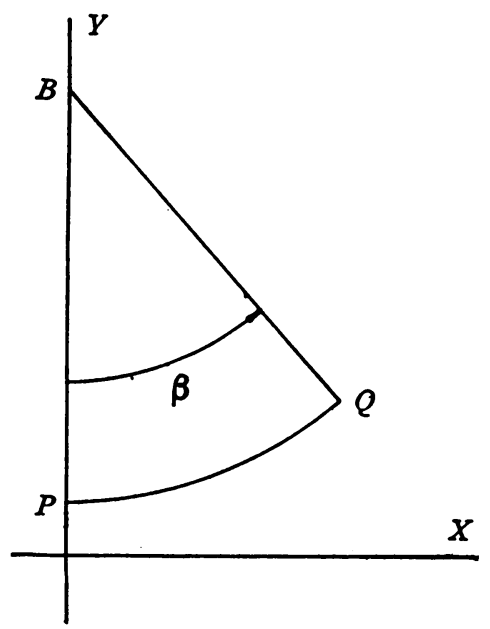

FIG. 2

$$
\lim \frac{b-y}{x}=\cot \beta, \quad \quad \text { for } Q \rightarrow B
$$

Then we have

$$
\lim \psi_{b}^{+}(x, y)=-S_{p-1} \beta, \quad \text { for } Q \rightarrow B .
$$

In fact, since by (32) and (35), $\psi_{b}^{+}\left(0, y_{P}\right)=0$ for $y_{P}<b$, we have, by (6),

$$
\psi_{b}^{+}(Q)=\int_{P Q} y^{p} \frac{\partial \phi_{b}}{\partial n} d s,
$$

the integral being taken along the circular arc $P Q$ of small radius $\epsilon$ and with the center at $B$. Using (17), we have on this arc 


$$
\frac{\partial \phi_{b}}{\partial n}=-S_{p-1} \epsilon^{-1}(b y)^{-p / 2}+2^{-1} p S_{p-1} b^{-p / 2} y^{-p / 2-1} \log \epsilon \frac{\partial y}{\partial n}+R^{*}(x, y)
$$

where $R^{*}(x, y)$ is regular at the point $B$. Putting $s=\epsilon \sigma$, we obtain therefore, by (39),

$$
\lim \psi_{b}^{+}(Q)=-S_{p-1} b^{-p / 2} \lim \int_{0}^{\beta} y^{p / 2} d \sigma=-S_{p-1} \beta,
$$

which proves the equation (38).

Let us now use the integral representation of $\psi_{0}^{+}$, as given by (26). By Gauss-Legendre duplication theorem, we have, for $q \neq 0$,

$$
2^{2 q-1} \Gamma(q) \Gamma(q+1 / 2)=\pi^{1 / 2} \Gamma(2 q),
$$

which shows, by (10) and (20), that

$$
\beta S_{p-1} C_{q}^{-1}=\pi^{-1} \beta, \quad \text { for } q>-1 / 2 .
$$

In this way we obtain, by (26) and (40), the fundamental formula

$$
\lim y^{q+1} \int_{0}^{\infty} e^{-x t} J_{q+1}(y t) J_{q}(b t) d t=\pi^{-1} \beta b^{q}
$$

which holds, under the condition (37), for $0 \leqq \beta \leqq \pi, x \geqq 0$. (A similar formula holds, obviously, for $x \leqq 0$.)

By putting $\beta=\pi$, we obtain the limit of $\psi_{b}^{+}(0, y)$ for $y=b+0$. Since, for $y>b, \psi_{b}^{+}(0, y)$ is identically equal to $K$, we have therefore, by $(40)$,

$$
K=-\pi S_{p-1} \text {. }
$$

Using this result, we obtain, by (28), (43) and (30),

$$
W_{b}(y)=b^{a} y^{-q-1}
$$

for $y>b$.

Let us now put $\beta=\pi / 2$. By the divergence theorem, the limit which we obtain in this case by $(40)$ is equal to the $\lim \psi_{b}^{+}(x, b)$ for $x$ tending to zero, namely $-S_{p-1} \pi / 2$. Since the integral (30) is convergent for $y=b$, we have therefore, by (43),

$$
W_{b}(b)=b^{-1 / 2} \text {. }
$$

Summarizing, we see that (43) implies, as a special case, the famous formulas of Weber and Schaftheitlin:

$$
\int_{0}^{\infty} J_{q+1}(y t) J_{q}(b t) d t=\left\{\begin{array}{lr}
0 & \text { for } 0 \leqq y<b, \\
(2 b)^{-1} & \text { for } y=b, \\
b^{q} y^{-q-1} & \text { for } y>b .
\end{array}\right.
$$


For any value of $\beta$, the fundamental formula (43) expresses the fact that $\psi_{b}^{+}(x, y)$ behaves in the neighborhood of $B$ like the angle $\beta$. The same is obviously true for $\psi_{b}^{-}$. The jump $\psi_{b}^{+}-\psi_{b}^{-}$across the segment $b<y<\infty$ is equal to $2 K$. This quantity, which, by (44), is equal to $-2 \pi S_{p-1}$, is also the period of the many-valued stream function $\psi_{b}(x, y)$ on a clockwise circuit around $B$.

7. The fundamental solution of the equation for the stream function. The easiest way to obtain a fundamental solution of the equation (3) is to use the identity

$$
y^{p+1}\left(f_{x x}+f_{y y}+(p+2) y^{-1} f_{y}\right)=g_{x x}+g_{y y}-p y^{-1} g_{y},
$$

where

$$
g(x, y)=y^{p+1} f(x, y) .
$$

This curious identity, which is valid for all values of $p$, shows that a potential $\phi$ in a space of $p+4$ dimensions generates a stream function $\psi$ in a space of $n=p+2$ dimensions. We have therefore, by (9); the following fundamental solution of (3)

$$
\Psi_{b}(x, y)=S_{p-1} y^{p+1} \int_{0}^{\pi}\left(b^{2}+x^{2}+y^{2}-2 b y \cos \alpha\right)^{-p / 2-1} \sin ^{p+1} \alpha d \alpha .
$$

Using (19), we can replace (50) by the equivalent formula

$$
\Psi_{b}(x, y)=C_{q+1} b^{-q-1} y^{q+1} \int_{0}^{\infty} e^{-|x| t} J_{q+1}(y t) J_{q+1}(b t) d t .
$$

The corresponding potential $\bar{\phi}_{b}$ is many-valued and is given, for $x>0$, by

$$
\Phi_{b}^{+}(x, y)=-C_{q+1} b^{-q-1} y^{-q} \int_{0}^{\infty} e^{-x t} J_{q}(y t) J_{q+1}(b t) d t .
$$

All the preceding formulas will be employed in another paper on the generalized theory of analytic functions, which is essentially based on the singular functions corresponding to the real and imaginary part of $\log z$ in the classical case.

8. Extension of the previous results. In this section we assume that $n=p+2$ is greater than or equal to 2 . The case $p=0$ corresponds to the twodimensional space.

The equations (1) admit, for $p \geqq 0$, the particular solutions

$$
\begin{aligned}
& \phi^{(1)}(x, y)=e^{-x t} y^{-\mu} J_{\mu}(y t), \\
& \psi^{(1)}(x, y)=-e^{-x t} y^{\mu+1} J_{\mu+1}(y t),
\end{aligned}
$$

where 


$$
\mu=(p-1) / 2 \geqq-1 / 2 .
$$

These solutions have already been used previously for $\mu>-1 / 2$.

Another pair of solutions of (1) is given by

$$
\begin{aligned}
& \phi^{(2)}(x, y)=e^{-x t} y^{y} J_{\nu}(y t), \\
& \psi^{(2)}(x, y)=e^{-x t} y^{1-y} J_{v-1}(y t),
\end{aligned}
$$

where

$$
\nu=(1-p) / 2 \leqq 1 / 2 .
$$

Using these functions we see that

$$
\begin{gathered}
\phi(x, y)=y^{\nu} \int_{0}^{\infty} e^{-|x| t} J_{\nu}(y t) J_{\nu}(b t) d t, \\
\psi^{+}(x, y)=y^{1-\nu} \int_{0}^{\infty} e^{-x t} J_{\nu-1}(y t) J_{\nu}(b t) d t
\end{gathered}
$$

are, for $x \geqq 0, y \geqq 0$, a pair of solutions of (1) for

$$
0<\nu \leqq 1 / 2 \text {. }
$$

(This inequality is equivalent to $0 \leqq p<1$ and $2 \leqq n<3$.)

By comparing (59) with (19) we see that $\phi$ has, for $y>0$, only a logarithmic singularity at the point $B: x=0, y=b$. Moreover, $\partial \phi / \partial y$ becomes, for $\nu \neq 1 / 2$, infinite like $y^{2 v-1}$ (that is, like $y^{-p}$ ) for $y$ tending to zero. On the $y$ axis, $\partial \phi / \partial x=0$, except at $B$.

From (1) we see that $\psi^{+}(x, y)$ has only one singularity, namely at $B$, and that $\psi^{+}(0, y)$ takes constant values $m$ and $M$ on the segments $0 \leqq y<b$ and $b<y$ respectively. On the $x$ axis, $\psi^{+}$and its derivatives remain finite and continuous. We find easily, in the same way as for $\psi_{b}^{+}$, that $\psi^{+}$is a branch of a many-valued function $\psi(x, y)$ and that

$$
\lim \psi^{+}(Q)=m-\pi^{-1} \beta b^{-\nu}
$$

for $Q$ tending to $B$

along the segment $Q B$ (see Fig. 2). Taking $\beta=\pi$, we have

$$
\psi^{+}(Q)=M,
$$

so that (62) yields

$$
M=m-b^{-p} .
$$

In order to determine $m$, which as we know is equal to $\psi^{+}(0,0)$, we first compute, for $x>0$,

$$
\psi^{+}(x, 0)=2^{1-\nu} \Gamma^{-1}(\nu) \int_{0}^{\infty} e^{-x t} J,(b t) t^{\nu-1} d t
$$


By differentiation with respect to $x$ we obtain

$$
\frac{\partial \psi^{+}(x, 0)}{\partial x}=-2^{1-\nu} \Gamma^{-1}(\nu) \int_{0}^{\infty} e^{-x t} J_{\nu}(b t) t^{\nu} d t .
$$

By comparing this expression with (22) and (23) we see that the right-hand side is, up to a constant factor, equal to $\left(x^{2}+b^{2}\right)^{->-1 / 2}$. Since, by $(65), \psi^{+}(x, 0)$ vanishes for $x=+\infty$, we easily find by an integration with respect to $x$ and by letting $x \rightarrow 0$,

$$
m=\psi^{+}(0,0)=b^{-\nu}
$$

so that, by (64),

$$
M=0 .
$$

In this way we obtain the following formulas

$$
\psi^{+}(0, y)=\left\{\begin{array}{lr}
b^{-\nu} & \text { for } 0 \leqq y<b, \\
2^{-1} b^{-\nu} & \text { for } y=b, \\
0 & \text { for } y>b,
\end{array}\right.
$$

corresponding to the cases $\beta=0, \pi / 2$, and $\pi$ in (62).

This result is equivalent to the formulas of Weber-Schafheitlin

$$
\int_{0}^{\infty} J_{\nu-1}(y t) J_{\nu}(b t) d t=\left\{\begin{array}{lr}
y^{\nu-1} b^{-\nu} & \text { for } 0<y<b, \\
(2 b)^{-1} & \text { for } y=b, \\
0 & \text { for } y>b .
\end{array}\right.
$$

These formulas have been established here only for $0<\nu \leqq 1 / 2$. However, from (47) it follows that they are actually valid for the range

$$
0<\nu<\infty,
$$

that is, for all values of $\nu$ for which the integrals (70) are convergent.

9. Dirichlet's discontinuous integral. The case $\nu=1 / 2(n=2)$ deserves a special mention. Putting in (60) $\nu=1 / 2$ and multiplying by the constant factor $\pi b^{1 / 2}$ we obtain, for $x \geqq 0$, the stream function

$$
\alpha(x, y)=2 \int_{0}^{\infty} t^{-1} e^{-x t} \cos (y t) \sin (b t) d t
$$

which can easily be computed explicitly either by the use of elementary formulas or by a suitable application of our identification principle. The result is that $\alpha(x, y)$ is the angle at the point $(x, y)$ subtended by the segment $(-b, b)$ of the $y$ axis $(0 \leqq \alpha \leqq \pi)$. The function $\alpha(x, y)$ can be interpreted as the stream function of axial doublets distributed along the segment $(-b, b)$. Putting $x=0$ and dividing by $\pi$, we obtain Dirichlet's discontinuous integral 
(73)

$$
2 \pi^{-1} \int_{0}^{\infty} t^{-1} \cos (y t) \sin (b t) d t=\pi^{-1} \alpha(0, y),
$$

for which we have found here an interpretation in terms of potential theory and many-valued functions $\left({ }^{6}\right)$.

Carnegie Institute of Technology, Pittsburgh, Pa.

(6) Added in proof (March 2, 1948). For further results and literature see: 1 . A. Weinstein, Quarterly of Applied Mathematics vol. 5 (1948) pp. 429-444. 2. A. Weinstein, Courant Anniversary Volume, Interscience, New York, 1948, pp. 451-460, where a more general class of equations is discussed, first introduced by Bers and Gelbart, loc. cit. footnote 3. The basic facts on many-valued associated functions referred to in footnote 5 have since been used by S. Bergman, Duke Math. J. vol. 14 (1947) pp. 349-366. 